\title{
Pressure Dependence of Elastic Constants in Zinc-Blende III-N and Their Influence on the Light Emission in Nitride Heterostructures
}

\author{
S.P. EEPKOWSKI ${ }^{a, *}$ AND J.A. MAJEWSKI ${ }^{b}$ \\ ${ }^{a}$ Unipress, High Pressure Research Center \\ Polish Academy of Sciences \\ Sokołowska 29, 01-142 Warszawa, Poland
}

${ }^{b}$ Walter Schottky Institute, Technische Universität München, Garching, Germany

\begin{abstract}
We studied the nonlinear elasticity effects for the case of III-N compounds. Particularly, we determined the pressure dependences of elastic constants, in zinc-blende InN, GaN, and AlN by performing ab initio calculations in the framework of plane-wave pseudopotential implementation of the density-functional theory. We found significant and almost linear increase in $C_{11}, C_{12}$ with pressure for considered nitrides compounds. Much weaker dependence on pressure was observed for $C_{44}$. We also discussed pressure dependences of two-dimensional Poisson's ratio and elastic anisotropy coefficient. Finally, we showed that the pressure dependence of elastic constants results in significant reduction of the pressure coefficient of the energy emission in cubic InGaN/GaN quantum wells and essentially improves the agreement between experimental and theoretical values.
\end{abstract}

PACS numbers: 62.20.Dc, 62.50.+p

\section{Introduction}

The electronic and optical properties of semiconductor heterostructures depend crucially on the strain arising from the lattice mismatch. Commonly, the strain effects in quantum structures, i.e. quantum wells (QWs), wires or dots (QDs), are described using the standard elasticity theory with elastic constants

*corresponding author; e-mail: slawek@unipress.waw.pl 
independent of the strain (so-called linear theory). Nevertheless, there are circumstances where this simple approach is insufficient.

Nonlinear elastic properties of GaAs and In As have recently attracted significant attention. First, Frogley et al. proposed that pressure dependences of elastic constants in GaAs and InAs are required to explain anomalously small pressure coefficient of band gap $\left(\mathrm{d} E_{\mathrm{G}} / \mathrm{d} P\right)$ in strained InGaAs layers [1]. They showed that the main contribution, responsible for drastic reduction of $\mathrm{d} E_{\mathrm{G}} / \mathrm{d} P$ in biaxial strained layers of InAs, came from the pressure dependence of a two-dimensional Poisson ratio, $\nu_{2 \mathrm{D}}(P)$, defined for zinc-blende structure as $2 C_{12} / C_{11}$. Second, Ellaway et al. calculated pressure dependences of elastic constants for InAs and discussed their influence on the properties of InAs/GaAs QDs [2,3]. They noticed that hydrostatic strain component in the InAs/GaAs QDs is significantly overestimated by calculations based on linear theory of elasticity. Introducing pressure dependence of elastic constant for InAs leads to reduction of the hydrostatic strain by about $16 \%$ [3]. For InAs, pressure dependence of an elastic anisotropy coefficient, defined as $\alpha=\left(C_{11}-C_{12}\right) / 2 C_{44}$ was also calculated [2]. The $\alpha$ coefficient describes degree of anisotropy of elastic properties in cubic crystals. Its knowledge is important for modeling of lateral ordering of QDs [4]. For InAs, the dependence of $\alpha$ on hydrostatic pressure was found to be rather weak, which led to the conclusion that the degree of elastic anisotropy in InAs QDs is approximately the same as in relaxed InAs layers, although high hydrostatic strain is present in the QDs [2].

For the case of III-N compounds, the nonlinear elasticity effects have not been systematically studied yet. A pioneering paper in this field was published by Kato and Hama who calculated the pressure dependence of elastic compliances for wurtzite AIN [5]. Recently, Vaschenko et al. have used results of these calculations to estimate the influence of the nonlinear elasticity on pressure coefficients of the light emission $\left(\mathrm{d} E_{\mathrm{E}} / \mathrm{d} P\right)$ in hexagonal $\mathrm{AlGaN} / \mathrm{GaN}$ QWs [6]. However, in hexagonal $\mathrm{AlGaN} / \mathrm{GaN}$ QWs, the magnitude of $\mathrm{d} E_{\mathrm{E}} / \mathrm{d} P$ strongly depends on the piezoelectric field which also shows nonlinear dependence on strain $[7,8]$. For this reason, the influence of the nonlinear elastic effects on $\mathrm{d} E_{\mathrm{E}} / \mathrm{d} P$ in hexagonal quantum structures is difficult to verify. Much better opportunity to study the nonlinear elastic effects is offered by pressure experiments done on [001] cubic InGaN/GaN QWs, since the piezoelectric field is absent in these structures due to symmetry. The experimental values of $\mathrm{d} E_{\mathrm{E}} / \mathrm{d} P$ for cubic $\operatorname{In}_{0.1} \mathrm{Ga}_{0.9} \mathrm{~N} / \mathrm{GaN}$ QWs with different QW widths were reported in Ref. [9]. They were compared with theoretical results obtained within a model based on the linear elastic theory [9]. Significant disagreement between experimental and theoretical results was found and attributed to the presence of non-uniform distribution of indium in the QWs [9].

In this work we study the nonlinear elasticity effects in nitride binary compounds. Particularly, we have determined the pressure dependences of elastic constants, $C_{11}, C_{12}$, and $C_{44}$, in zinc-blende $\operatorname{InN}, \mathrm{GaN}$, and AlN by performing 
$a b$ initio calculations in the framework of plane-wave pseudopotential implementation of the density-functional theory $[10,11]$. Next, we discuss the pressure dependence of both earlier mentioned coefficients $\nu_{2 \mathrm{D}}(P)$ and $\alpha(P)$ for the case of nitrides. Finally, we show that the knowledge of the pressure derivatives of the elastic constants is essential for the calculations of $\mathrm{d} E_{\mathrm{E}} / \mathrm{d} P$ in cubic InGaN/GaN QWs. We observe that taking into account the pressure dependence of elastic constants leads to significant reduction of the $\mathrm{d} E_{\mathrm{E}} / \mathrm{d} P$ in cubic InGaN/GaN QWs as compared to results obtained with linear elastic theory and decisively increases the agreement between theory and experiment.

The paper is organized as follows. In Sec. 2 we determine the dependence of elastic constants on hydrostatic pressure in zinc-blende $\mathrm{GaN}, \mathrm{InN}$, and AIN. In Sec. 3 the pressure dependence of $\nu_{2 \mathrm{D}}(P)$ and $\alpha(P)$ is discussed. The influence of the nonlinear elasticity in zinc-blende $\mathrm{GaN}$ and $\mathrm{InN}$ on $\mathrm{d} E_{\mathrm{E}} / \mathrm{d} P$ in cubic $\mathrm{InGaN} / \mathrm{GaN} \mathrm{QWs}$ is shown in Sec. 4. Conclusions are presented in the last section.

\section{Pressure dependence of elastic constants}

The pressure dependence of elastic constants, $C_{11}, C_{12}$, and $C_{44}$ in zinc-blende $\mathrm{GaN}, \mathrm{InN}$, and AlN has been determined by carrying out the total energy calculations based on a plane-wave pseudopotential implementation of the density-functional theory [10]. The numerical computations have been performed with the VASP package [11].

Following the arguments of Ref. [12], the pressure dependent elastic constants are calculated as

$$
C_{\alpha \beta}(P)=\frac{1}{V(P)} \frac{\partial^{2} E_{\text {tot }}(V(P))}{\partial \varepsilon_{\alpha} \partial \varepsilon_{\beta}},
$$

where $E_{\text {tot }}(V(P))$ is the total energy per unit cell, $V(P)$ is the unit cell volume at given pressure $P$, which is found by solving $P=-\partial E_{\text {tot }} / \partial V$, and $\varepsilon_{\alpha}, \varepsilon_{\beta}$ are the elements of the infinitesimal Lagrangian strain tensor.

In Figs. $1 \mathrm{a}-\mathrm{c}$ we present the elastic constants $C_{11}, C_{12}$, and $C_{44}$ in zinc-blende $\mathrm{GaN}, \mathrm{InN}$, and $\mathrm{AlN}$ as a function of hydrostatic pressure. One can see that $C_{11}, C_{12}$ vary almost linearly with pressure, $C_{\alpha \beta}(P)=C_{\alpha \beta}+C_{\alpha \beta}^{\prime} P$, with $(\alpha \beta)=(11)$, (12). The $C_{44}$ elastic constants show much weaker dependence on pressure and slightly sublinear character. At ambient pressure, we have obtained $C_{11}=252 \mathrm{GPa}$, $C_{12}=131 \mathrm{GPa}$, and $C_{44}=146 \mathrm{GPa}$ for $\mathrm{GaN}, C_{11}=149 \mathrm{GPa}, C_{12}=94 \mathrm{GPa}$, and $C_{44}=77 \mathrm{GPa}$ for InN, and $C_{11}=267 \mathrm{GPa}, C_{12}=141 \mathrm{GPa}$, and $C_{44}=172 \mathrm{GPa}$ for $A l N$, respectively. These results are in good agreement with previous calculations $[13,14]$ and experiments [15]. For further calculations, it is convenient to determine the linear coefficients $C_{11}^{\prime}$ and $C_{12}^{\prime}$. These pressure derivatives of elastic constants are equal to $C_{11}^{\prime}=3.63$ and $C_{12}^{\prime}=3.18$ for $\mathrm{GaN}, C_{11}^{\prime}=3.75$ and $C_{12}^{\prime}=3.81$ for InN and $C_{11}^{\prime}=3.77$ and $C_{12}^{\prime}=3.12$ for AlN. From these results, one can estimate the derivative of bulk modulus, $B_{0}^{\prime}$, which equals to $3.33,3.79$, and 

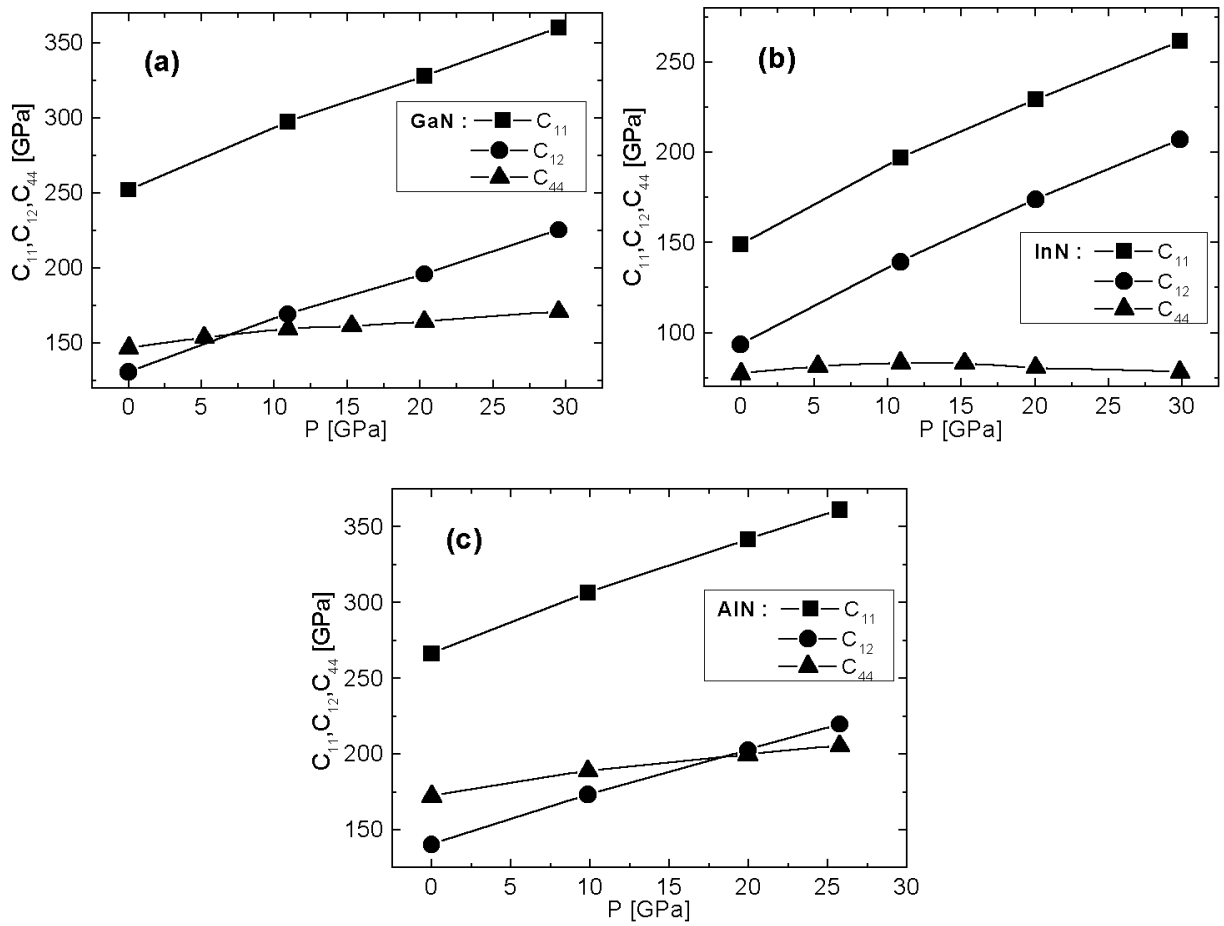

Fig. 1. Elastic constants $C_{11}$ (squares), $C_{12}$ (dots), and $C_{44}$ (triangles) for (a) zinc-blende $\mathrm{GaN}$, (b) zinc-blende $\mathrm{InN}$, and (c) zinc-blende AIN plotted as a function of hydrostatic pressure. Solid lines are added to guide the eye.

3.34 for GaN, InN, and AlN, respectively, and agrees very well with values of $B_{0}^{\prime}$ obtained from the Murnaghan equation of state [12]. Again, the values of $B_{0}^{\prime}$ for $\mathrm{GaN}, \mathrm{InN}$, and AlN agree well with previous calculations and experiments $[15,16]$.

\section{Two-dimensional Poisson's ratio and elastic anisotropy coefficient}

Having determined the pressure dependence of the elastic constants in cubic nitrides, we proceed to discussion of $\nu_{2 \mathrm{D}}(P)$ and $\alpha(P)$ for these compounds. In Fig. 2, $\nu_{2 \mathrm{D}}(P)$ is plotted for pressures up to $30 \mathrm{GPa}$. Similarly to the case $C_{11}(P)$ and $C_{12}(P)$, we observe that $\nu_{2 \mathrm{D}}(P)$ varies almost linearly with pressure, $\nu_{2 \mathrm{D}}(P)=$ $\nu_{2 \mathrm{D}}+\nu_{2 \mathrm{D}}^{\prime} P$. For InN, the increase in $\nu_{2 \mathrm{D}}(P)$ with pressure is significantly larger than for other two compounds. One can estimate values of the coefficient $\nu_{2 \mathrm{D}}^{\prime}$ which are equal to $0.0072,0.0111,0.0063 \mathrm{GPa}^{-1}$ for $\mathrm{GaN}, \mathrm{InN}$, and AlN, respectively. The knowledge of $\nu_{2 \mathrm{D}}^{\prime}$ is important since the pressure coefficient of the band gap in strain layers is significantly reduced by a term which is directly proportional to the product of $\nu_{2 \mathrm{D}}^{\prime}$ and initial biaxial strain [1]. Thus, one can regard $\nu_{2 \mathrm{D}}^{\prime}$ as a good measure of the sensitivity of $\mathrm{d} E_{\mathrm{G}} / \mathrm{d} P$ to the biaxial strain in a particular 


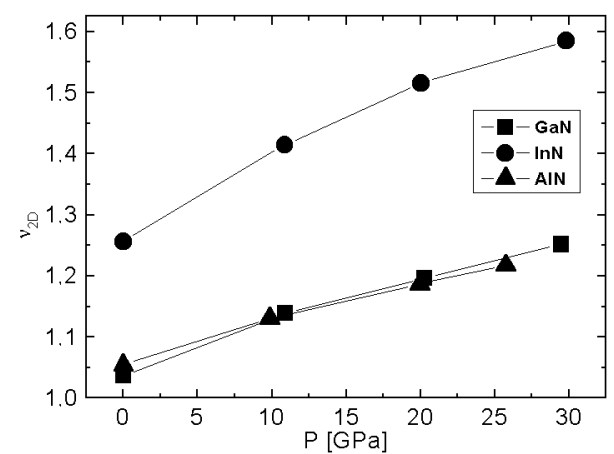

Fig. 2. Two-dimensional Poisson's ratio for zinc-blende GaN (squares), InN (circles), and AlN (triangles) plotted as a function of hydrostatic pressure. Solid lines are added to guide the eye.

material. Since $\nu_{2 \mathrm{D}}^{\prime}$ is significantly larger for InN than for GaN or AlN, one can expect that the biaxial strain affects more strongly $\mathrm{d} E_{\mathrm{G}} / \mathrm{d} P$ in strained $\mathrm{InN}$ or InGaN layers than in strained GaN or AlGaN structures.

In Fig. 3, we show the pressure dependence of $\alpha(P)$ for GaN, InN, and AlN, respectively. As we have mentioned in the introduction, $\alpha$ describes the degree of elastic anisotropy of a cubic crystal. For ideal isotropic crystal $\alpha$ is equal to 1 . Typically, $\alpha$ takes value of around 0.5 for zinc-blende semiconductors. Knowing the value of $\alpha$, one can estimate an error introduced in strain-stress calculations done using the isotropic elastic theory. The degree of elastic anisotropy is larger for InN and AlN than for GaN, as one can see in Fig. 3. Generally, we observe that $\alpha$ does not change significantly with pressure for all cubic nitrides, in accordance with physical intuition.

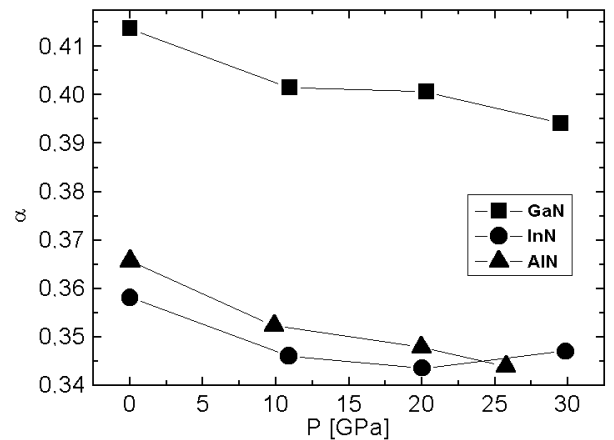

Fig. 3. Elastic anisotropy coefficient for zinc-blende GaN (squares), InN (circles), and AIN (triangles) plotted as a function of hydrostatic pressure. Solid lines are added to guide the eye. 


\section{Pressure coefficients of the light emission in cubic InGaN/GaN QWs}

In this section we show the influence of nonlinear elasticity (i.e. the pressure dependence of elastic constants) on $\mathrm{d} E_{\mathrm{E}} / \mathrm{d} P$ in c-InGaN/GaN QWs. To do so, we calculate energetic positions of the lowest electron and the highest hole states in the QWs for various hydrostatic pressures employing $\boldsymbol{k} \cdot \boldsymbol{p}$ envelope function theory. The shift of the band edges caused by the hydrostatic and biaxial strain has been accounted for within the deformation potential theory. For each pressure $P$, the value of the biaxial strain in the InGaN QW has been determined from the Hook law following Ref. [17]. In this step the pressure dependent elastic constants for InGaN alloy have been used (we use Vegard-like law i.e. $C_{\alpha \beta}^{\mathrm{InGaN}}(P, x)=$ $(1-x) C_{\alpha \beta}^{\mathrm{GaN}}(P)+x C_{\alpha \beta}^{\operatorname{InN}}(P)$ with $\left.(\alpha \beta)=(11),(12)\right)$. Further, for $\operatorname{In}_{x} \mathrm{Ga}_{1-x} \mathrm{~N}$ alloy, linear dependence of the shear deformation potential $b$ on In concentration $x$ has been assumed [18], whereas nonlinear $x$-dependence has been used for the energy gap hydrostatic deformation potential $a_{\mathrm{g}}$ [19]. Parameters of the $\boldsymbol{k} \cdot \boldsymbol{p}$ matrix for c-InGaN have been taken from Refs. [20] and [21], however the pressure dependence of the conduction band effective mass has been also included [22].

In Fig. 4, we show theoretical and experimental [9] values of $\mathrm{d} E_{\mathrm{E}} / \mathrm{d} P$ for c- $\operatorname{In}_{0.1} \mathrm{Ga}_{0.9} N / G a N$ QWs as a function of QW width. For comparison theoretical values of $\mathrm{d} E_{\mathrm{E}} / \mathrm{d} P$ calculated using linear elastic theory [9] are also given. One can see that application of the nonlinear elastic theory results in significant reduction of $\mathrm{d} E_{\mathrm{E}} / \mathrm{d} P$ in $\mathrm{c}-\mathrm{In}_{0.1} \mathrm{Ga}_{0.9} \mathrm{~N} / \mathrm{GaN} \mathrm{QWs}$ by about $4 \mathrm{meV} / \mathrm{GPa}$. This result is consistent with anomalous $10 \%$ reduction of $\mathrm{d} E_{\mathrm{E}} / \mathrm{d} P$ found earlier in strained $\mathrm{In}_{0.1} \mathrm{Ga}_{0.9}$ As layers and explained by the nonlinear elastic theory [1]. As it is seen

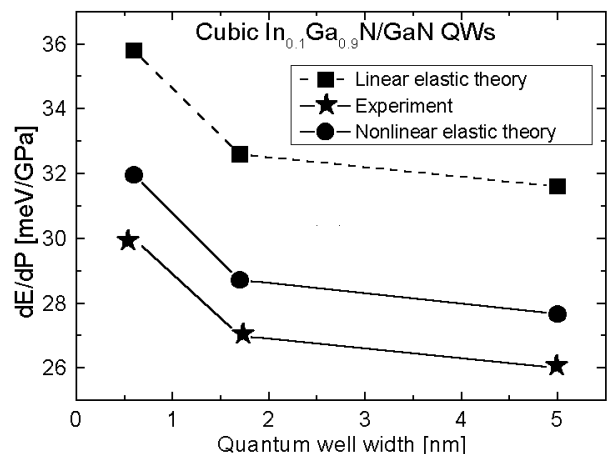

Fig. 4. Pressure coefficients of emission energies $\left(\mathrm{d} E_{\mathrm{E}} / \mathrm{d} P\right)$ from cubic $\mathrm{In}_{0.1} \mathrm{Ga}_{0.9} \mathrm{~N} / \mathrm{GaN}$ QWs as a function of $\mathrm{QW}$ width. Stars represent the experimental values of $\mathrm{d} E_{\mathrm{E}} / \mathrm{d} P$, taken from Ref. [9]. Squares correspond to theoretical values of $\mathrm{d} E_{\mathrm{E}} / \mathrm{d} P$ obtained using linear elastic theory (also taken from Ref. [9]). Circles correspond to magnitudes of $\mathrm{d} E_{\mathrm{E}} / \mathrm{d} P$ calculated using nonlinear elastic theory (this work). Solid and dashed lines are added to guide the eye. 
from Fig. 4, when the nonlinear elastic theory is used, the agreement between experimental and theoretical values of $\mathrm{d} E_{\mathrm{e}} / \mathrm{d} P$ in $c-\mathrm{In}_{0.1} \mathrm{Ga}_{0.9} \mathrm{~N} / \mathrm{GaN} \mathrm{QWs}$ is significantly improved. This clearly demonstrates that the pressure dependence of the elastic constants plays crucial role in quantitative explanation of the observed reduction in the pressure coefficient of the light emission in c-InGaN/GaN quantum wells. However, the issue of the role played by the indium fluctuations in the QWs in reduction of the $\mathrm{d} E_{\mathrm{E}} / \mathrm{d} P$ remains open and requires further experimental and theoretical studies.

\section{Conclusions}

In summary, we have calculated the pressure dependence of elastic constants in zinc-blende GaN, InN, and AIN. Significant and almost linear increase in $C_{11}, C_{12}$ with the hydrostatic pressure has been found for considered nitrides compounds. Much weaker dependence on pressure has been found for $C_{44}$. For all cubic nitrides, Poisson's ratio exhibits linear dependence on pressure (with the strongest effect in the case of $\mathrm{InN}$ ), whereas the elastic anisotropy coefficient does not show significant pressure dependence. Finally, we have studied the influence of nonlinear elastic effects on the pressure coefficients of light emission $\left(\mathrm{d} E_{\mathrm{E}} / \mathrm{d} P\right)$ in c-InGaN/GaN QWs. We have demonstrated that pressure dependence of elastic constants results in significant reduction of the $\mathrm{d} E_{\mathrm{E}} / \mathrm{d} P$ in $c-I n G a N / G a N ~ Q W s$ as compared to results obtained within linear elastic theory. This reduction considerably increases the agreement between experimental and theoretical values of $\mathrm{d} E_{\mathrm{E}} / \mathrm{d} P$ obtained for $c-\operatorname{In}_{0.1} \mathrm{Ga}_{0.9} \mathrm{~N} / \mathrm{GaN}$ QWs with various $\mathrm{QW}$ widths.

\section{Acknowledgments}

This work was supported by the State Committee for Scientific Research (Poland), project No. 4T11F 00825 and by the European Commission grant "Support for Centers of Excellence" No. ICA1-CT-2000-70005. The ab initio calculations of elastic constants were performed using computer facilities of Interdisciplinary Center for Mathematical and Computational Modeling of Warsaw University. One of us (J.A.M.) would like to acknowledge the hospitality he experienced during his visits to Unipress.

\section{References}

[1] M.D. Frogley, J.R. Downes, D.J. Dunstan, Phys. Rev. B 62, 13612 (2000).

[2] S.W. Ellaway, D.A. Faux, Phys. Status Solidi B 235, 437 (2003).

[3] S.W. Ellaway, D.A. Faux, J. Appl. Phys. 92, 3027 (2002).

[4] V. Holy, G. Springholz, M. Pinczolits, G. Baner, Phys. Rev. Lett. 83, 356 (1999).

[5] R. Kato, J. Hama, J. Phys., Condens. Matter 6, 7617 (1994). 
[6] G. Vaschenko, C.S. Menoni, D. Patel, C.N. Tome, B. Clausen, N.F. Gardner, J. Sun, W. Gotz, H.M. Ng, A.Y. Cho, Phys. Status Solidi B 235, 238 (2003).

[7] S.P. Łepkowski, H. Teisseyre, T. Suski, P. Perlin, N. Grandjean, J. Massies, Appl. Phys. Lett. 79, 1483 (2001).

[8] G. Vaschenko, D. Patel, C.S. Menoni, S. Keller, U.K. Mishra, S.P. DenBaars, Appl. Phys. Lett. 78, 640 (2001).

[9] T. Suski, H. Teisseyre, S.P. Łepkowski, P. Perlin, T. Kitamura, Y. Ishida, H. Okumura, S.F. Chichibu, Appl. Phys. Lett. 81, 232 (2002).

[10] W.E. Pickett, Comp. Phys. Rep. 9, 117 (1989).

[11] G. Kresse, J. Furthmuller, Phys. Rev. B 54, 11169 (1996).

[12] S. Bhagavantam, Crystal Symmetry and Physical Properties, Academic Press, London 1966, p. 147.

[13] A.F. Wright, J. Appl. Phys. 82, 2833 (1997).

[14] F. Grosse, J. Neugebauer, Phys. Rev. B 63, 085207 (2001).

[15] A. Polian, in: Properties, Processing and Applications of Gallium Nitride and Related Semiconductors, Eds. J.H. Edgar, S. Strite, I. Akasaki, H. Amano, C. Wetzel, Datareviews Series 3, INSPEC, London 1999, A1.3.

[16] S.-H. Wei, A. Zunger, Phys. Rev. B 60, 5404 (1999).

[17] E. Anastassakis, Phys. Rev. B 46, 13244 (1992).

[18] C.G. Van de Walle, J. Neugebauer, Appl. Phys. Lett. 70, 2577 (1997).

[19] P. Perlin, I. Gorczyca, T. Suski, P. Wisniewski, S. Łepkowski, N.E. Christensen, A. Svane, M. Hansen, S.P. DenBaars, B. Damilano, N. Grandjean, J. Massie, Phys. Rev. B 64, 115319 (2001).

[20] S.K. Pugh, D.J. Dugdale, S. Brand, R.A. Abram, Semicond. Sci. Technol. 14, 23 (1999).

[21] L.K. Teles, J. Furthmuller, L.M.R. Scalfaro, J.R. Leite, F. Bechstedt, Phys. Rev. $B$ 63, 085204-1 (2001).

[22] P. Perlin, W. Trzeciakowski, E. Litwin-Staszewska, J. Muszalski, M. Micovic, Semicond. Sci. Technol. 9, 2239 (1994). 